\title{
Impact of Fin Width on Tri-Gate GaN MOSHEMTs
}

\author{
Jun Ma ${ }^{\circledR}$, Giovanni Santoruvo ${ }^{\circledR}$, Luca Nela ${ }^{\oplus}$, Taifang Wang ${ }^{\circledR}$, and Elison Matioli॰ ${ }^{\circledR}$, Member, IEEE
}

\begin{abstract}
In this paper, we present a detailed investigation of the impact of fin width $\left(w_{\mathrm{fin}}\right)$ on tri-gate AIGaN/GaN metal-oxide-semiconductor high electron mobility transistors (MOSHEMTs). As $w_{\text {fin }}$ is reduced, the threshold voltage $\left(V_{\mathrm{TH}}\right)$ increases, which is due to the enhanced gate control (especially for $w_{\text {fin }}<200 \mathrm{~nm}$ ) thanks to the 3-D geometry of the tri-gate, and the reduced carrier concentration $\left(N_{\mathrm{s}}\right)$ caused by a more pronounced strain relaxation and sidewall depletion, as explored using Hall and capacitance-voltage $(C-V)$ measurements. The normallyOFF operation was achieved for $w_{\text {fin }}$ close to the sidewall depletion width $\left(w_{\text {dep }}\right)$ of $19.5 \mathrm{~nm}$, since the fin is depleted from its two sidewalls. The impact of $w_{\text {fin }}$ on oN-resistance $\left(\boldsymbol{R}_{\mathrm{ON}}\right)$ and current capability $\left(I_{\mathrm{D}, \mathrm{max}}\right)$ was also investigated, along with the influence of the effective source injection, the trench conduction and the filling factor (FF) on these key characteristics. The degradation caused by the tri-gate fin etching could be fully recovered by increasing the FF. Finally, we show that the tri-gate can reduce gate capacitance $\left(C_{\mathrm{G}}\right)$ and charge $\left(Q_{\mathrm{G}}\right)$ in normally-ON MOSHEMTs, depending on the design of the tri-gate and the gate voltage $\left(V_{\mathrm{G}}\right)$, leading to a smaller $R_{\mathrm{ON}} \cdot Q_{\mathrm{G}}$ product that is beneficial for high-frequency switching applications. The results in this paper offer insights into important phenomena in tri-gate GaN devices and are fundamental for the further advance of this technology.
\end{abstract}

Index Terms-Drain current, fin width, GaN, gate capacitance, threshold voltage, tri-gate.

\section{INTRODUCTION}

$\mathbf{T}$ RI-GATE technologies have recently attracted considerable attention for lateral $\mathrm{GaN}$ electronic devices [1]-[20], thanks to many advantages over conventional planar gates. First, the $V_{\mathrm{TH}}$ in tri-gate $\mathrm{GaN}$ metal-oxide-semiconductor high electron mobility transistors (MOSHEMTs) increases as $w_{\text {fin }}$ decreases, hence, the normally-OFF operation can be

Manuscript received February 22, 2019; revised April 18, 2019, May 30, 2019, and June 24, 2019; accepted June 26, 2019. This work was supported in part by the European Research Council through the European Union's H2020 Program/ERC under Grant Agreement 679425, and in part by the Swiss National Science Foundation under Grants PYAPP2_166901 and 200021_169362. The review of this paper was arranged by Editor B. K. Kaushik. (Corresponding authors: Jun Ma; Elison Matioli.)

The authors are with the Power and Wide-Band-Gap Electronics Research Laboratory (POWERlab), École polytechnique fédérale de Lausanne (EPFL), 1015 Lausanne, Switzerland (e-mail: jun.ma @epfl.ch; elison.matioli@epfl.ch).

Color versions of one or more of the figures in this paper are available online at http://ieeexplore.ieee.org.

Digital Object Identifier 10.1109/TED.2019.2925859

achieved by reducing $w_{\text {fin }}$ [21], by recessing the tri-gate [22], or with novel gate oxides such as $\mathrm{MgCaO}$ [23]. Meanwhile, the dependence of $V_{\mathrm{TH}}$ on $w_{\text {fin }}$ in tri-gated GaN heterostructures offers a lateral scheme to design and construct novel field plates (FPs) for high-voltage power devices [24]-[26], such as the slanted tri-gate, which resulted in power GaN-on-Si MOSHEMTs with state-of-the-art performance [24], and in power GaN-on-Si Schottky barrier diodes (SBDs) with record breakdown voltage $(-2000 \mathrm{~V}$ at $1 \mu \mathrm{A} / \mathrm{mm})$ and ultralow reverse leakage current $(5 \mathrm{nA} / \mathrm{mm}$ at $-650 \mathrm{~V})$ [25]. In addition, the tri-gates also offer a unique opportunity to control multiple parallel 2-dimensional-electron-gas (2DEG) channels, leading to novel multi-channel tri-gate devices with much reduced $R_{\mathrm{ON}}$ and enhanced current capability [22], [27], which are essential to enhance the energy efficiency and power density of GaN power and RF devices. Furthermore, the tri-gate can provide excellent linearity [28]-[32], high $g_{\mathrm{m}}$ [13], small subthreshold swing (SS) [19], high ON/OFFstate current ratio [7], [27], and diminished short channel effect [7], [22], [33], along with many other merits that are beneficial for switching and amplifying applications.

While most of these remarkable properties of the tri-gate depend on $w_{\text {fin }}$ (or the tri-gate width), the full impact of $w_{\text {fin }}$ on characteristics of tri-gate $\mathrm{GaN}$ transistors has not yet been well understood. A better comprehension of this key designing parameter is fundamental and highly desirable to unleash the full potential of the promising tri-gate technology.

In this paper, we present a detailed investigation of tri-gate GaN MOSHEMTs and reveal the impact of $w_{\text {fin }}$ on device characteristics such as $V_{\mathrm{TH}}, g_{\mathrm{m}}, I_{\mathrm{D}, \max }, R_{\mathrm{ON}}, C_{\mathrm{G}}$, and $Q_{\mathrm{G}}$. We explore the major reasons for the dependence of $V_{\mathrm{TH}}$ on $w_{\text {fin }}$, quantified the dependence of $N_{\mathrm{s}}$ on $w_{\text {fin }}$, and show that the sidewall depletion is critical to achieving normallyOFF tri-gate $\mathrm{GaN}$ transistors. In addition, we analyze the impact of $w_{\text {fin }}$ on $I_{\mathrm{D}, \max }$, and $R_{\mathrm{ON}}$, discuss the importance of effective source injection, trench conduction, and FF and present the potential of tri-gates in reducing $C_{\mathrm{G}}$ and $Q_{\mathrm{G}}$ for normally-ON GaN (MOS)HEMTs. These results provide important insights for optimizing tri-gate $\mathrm{GaN}$ devices and addressing the tradeoffs for different applications.

\section{DEVICE FABRICATION}

The $\mathrm{AlGaN} / \mathrm{GaN}$-on-Si epitaxy in this paper consisted of $3.75 \mu \mathrm{m}$ of the buffer, $0.3 \mu \mathrm{m}$ of undoped $\mathrm{GaN}$ (u-GaN)

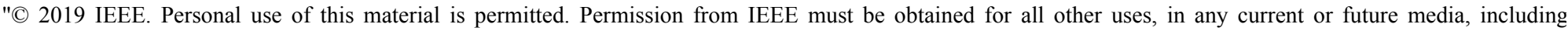

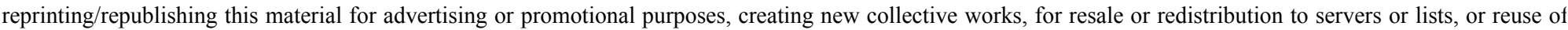
any copyrighted component of this work in other works. 

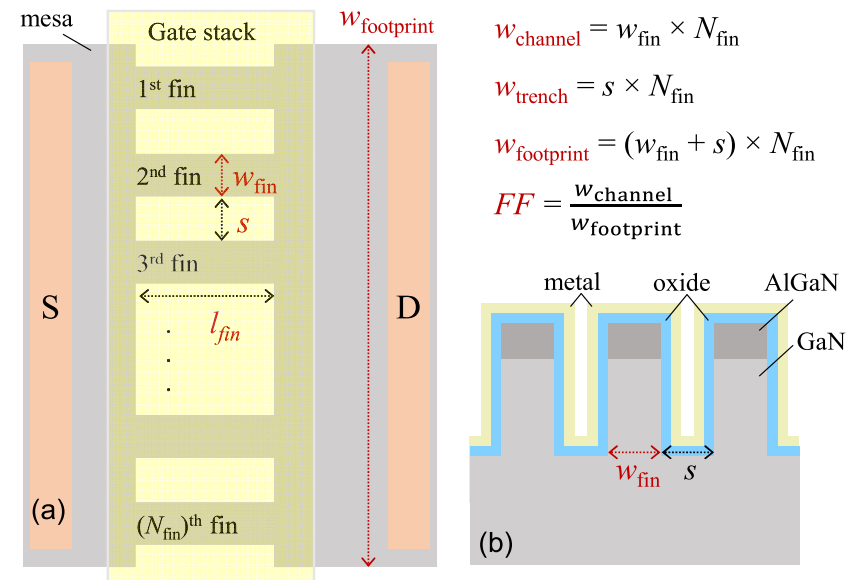

Fig. 1. (a) Top view schematic of a tri-gate AIGaN/GaN MOSHEMT. (b) Cross-sectional schematic of the tri-gate region. Important geometric parameters of the tri-gate regions are defined here, including the width of a fin $\left(w_{\text {fin }}\right)$, the width of a trench $(s)$, the number of fins in a given device $\left(N_{\text {fin }}\right)$, the length of the fins $\left(I_{\text {fin }}\right)$, the FF, the width of device footprint $\left(w_{\text {footprint }}\right)$, the effective channel width $\left(w_{\text {channel }}=w_{\text {fin }} \times N_{\text {fin }}\right)$, and the total width of the trenches $\left(w_{\text {trench }}=s \times N_{\text {fin }}\right)$.

channel, $23.5 \mathrm{~nm}$ of AlGaN barrier, and $2 \mathrm{~nm}$ of u-GaN cap layers. The sheet resistance was about $360 \Omega /$ sq, and the electron mobility was about $2000 \mathrm{~cm}^{2} \cdot \mathrm{V}^{-1} \mathrm{~s}^{-1}$. Three types of devices were fabricated and studied: MOSHEMTs and MOS capacitors with planar and tri-gate topologies, and nanoscale Hall crosses. The MOSHEMTs had different $w_{\text {fin }}$ but the same nominal FF of 50\% ( $s$ and $w_{\text {fin }}$ shown in Fig. 1 were about the same), along with the same width of the device footprint $\left(w_{\text {footprint }}=60 \mu \mathrm{m}\right)$. The fins were 700-nm long $\left(l_{\text {fin }}\right), 166-\mathrm{nm}$ high, and 2 and $11.3 \mu \mathrm{m}$ apart from the source and drain electrodes, respectively. The gate metal was $2.5-\mu \mathrm{m}$ long, covering the entire length of the fins and extending 0.5 and $1.3-\mu \mathrm{m}$ toward the source and drain electrodes, respectively. Details about the MOS capacitors and hall crosses are introduced later.

The device fabrication started with e-beam lithography to define the fins and mesa, which were etched using $\mathrm{Ar} / \mathrm{Cl}_{2}$-based inductively coupled plasma (ICP). Ohmic metals were then formed by lift-off and annealing processes, followed by $20-\mathrm{nm} \mathrm{Al}_{2} \mathrm{O}_{3}$ as the dielectric and $\mathrm{Ni} / \mathrm{Au}$ as the gate metals.

Crucial geometrical components of the fins are shown in Fig. 1. In addition to $w_{\text {fin }}, s$, and FF, we define the number of fins in a given device as $N_{\text {fin }}$, the effective channel width as $w_{\text {channel }}$, and the total width of the trenches as $w_{\text {trench }}$, which are important terms to understand the influence of tri-gates on device characteristics. All measured current values in this paper were normalized by $w_{\text {footprint }}$, and the standard deviation was determined from about ten devices of each type.

\section{Impact on Transfer Characteristics}

Transfer characteristics of tri-gate GaN MOSHEMTs are significantly impacted by $w_{\text {fin }}$. As shown in Fig. 2(a), $V_{\mathrm{TH}}$ increased and SS reduced as $w_{\text {fin }}$ was reduced. In planar MOSHEMTs, $V_{\mathrm{TH}}$ was $-7.43 \pm 0.07 \mathrm{~V}$ and SS was $98 \pm 14 \mathrm{mV} / \mathrm{dec}$, which were improved by the tri-gate with
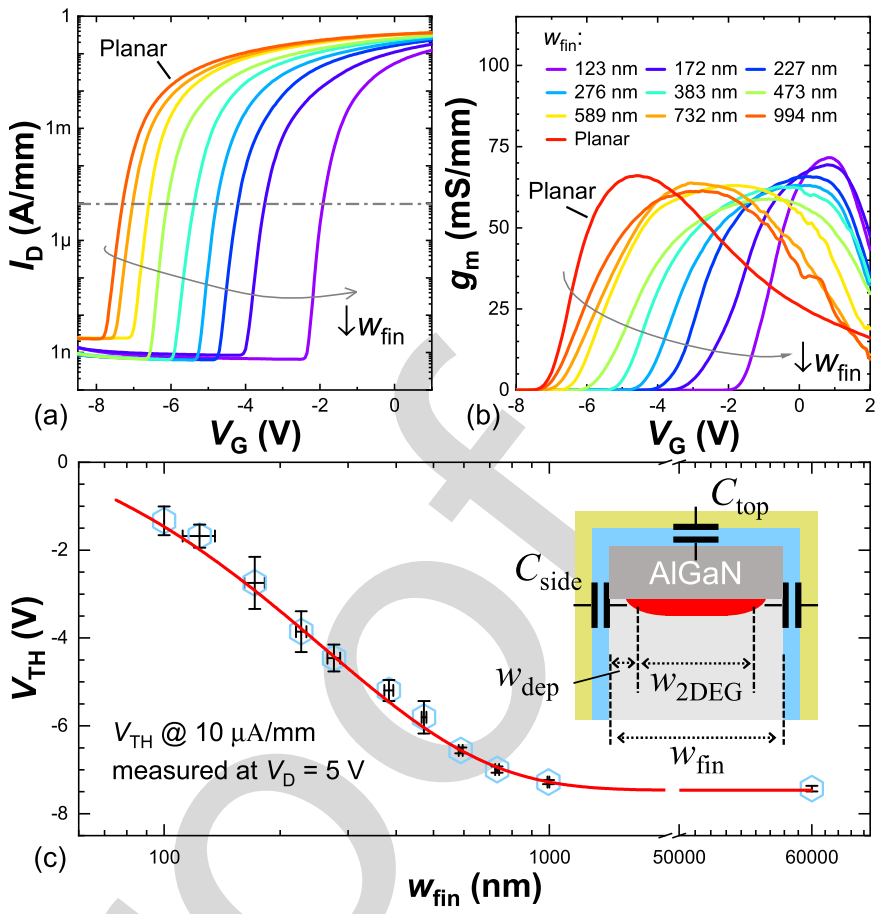

Fig. 2. Average (a) $I_{D}-V_{G}$ and (b) $g_{m}-V_{G}$ characteristics in tri-gate AIGaN/GaN MOSHEMTs, measured at $V_{\mathrm{D}}=5 \mathrm{~V}$. The gate leakage current of all these devices was below $0.1 \mathrm{nA} / \mathrm{mm}$ at $V_{\mathrm{G}}=-8.5 \mathrm{~V}$. (c) Summarized dependence of $V_{\mathrm{TH}}$ on $w_{\text {fin }}$, in which $w_{\text {fin }}$ was measured by scanning electron microscopy (SEM) and $V_{\mathrm{TH}}$ was defined at $10 \mu \mathrm{A} / \mathrm{mm}$. The inset in (c) illustrates the effect of sidewall depletion in distributing the 2DEGs across a fin.

$w_{\text {fin }}$ of $123 \mathrm{~nm}$ to $-1.68 \pm 0.26 \mathrm{~V}$ and $83 \pm 5 \mathrm{mV} / \mathrm{dec}$, respectively. These results are consistent with other works in the literature [1], [7], [22] and indicate a better electrostatic control using the tri-gate.

The enhanced control in tri-gate $\mathrm{GaN}$ transistors, however, does not necessarily improve the $g_{\mathrm{m}}$ as usually expected. As shown in Fig. 2(b), $g_{\mathrm{m}}$ first reduced to $59.1 \pm 2 \mathrm{mS} / \mathrm{mm}$ when $w_{\text {fin }}$ was decreased to $473 \mathrm{~nm}$, which is smaller than in planar devices $(66.1 \pm 2 \mathrm{mS} / \mathrm{mm})$, but then increased to $71.9 \pm 2 \mathrm{mS} / \mathrm{mm}$ at $w_{\text {fin }}$ of $123 \mathrm{~nm}$. This phenomenon is mainly caused by the tradeoff between the electric conductivity of the channel and the electrostatic control of the tri-gate. By replacing the planar gate with wide tri-gates, the electrical conductivity of the device was degraded due to the fin etching, along with limited enhancement in electrostatic control, resulting in a decreased $g_{\mathrm{m}}$. By narrowing the fins further, the trigate control was greatly enhanced, overcoming the degradation in conductivity and resulting in a higher $g_{\mathrm{m}}$.

The observation of the smaller $g_{\mathrm{m}}$ in tri-gate $\left(w_{\text {fin }} \geq 276 \mathrm{~nm}\right)$ than in planar devices in this paper differs from many prior arts, in which an enhanced $g_{\mathrm{m}}$ is usually reported. This is a result of the difference in normalization methods for the $I_{\mathrm{D}}$ in this paper and the previous reports. While often in the literature, the $I_{\mathrm{D}}$ is normalized by $w_{\text {channel }}\left(w_{\text {fin }} \times N_{\text {fin }}\right)$ for tri-gate GaN devices, i.e., the width of the $2 D E G$ in the tri-gate region, and in this paper, it was normalized by $w_{\text {footprint }}\left(\left(w_{\text {fin }}+s\right) \times N_{\text {fin }}\right)$. The former better reveals the characteristics of the fins locally,

"C 2019 IEEE. Personal use of this material is permitted. Permission from IEEE must be obtained for all other uses, in any current or future media, including reprinting/republishing this material for advertising or promotional purposes, creating new collective works, for resale or redistribution to servers or lists, or reuse of any copyrighted component of this work in other works. 
but it may underestimate the effect of the access regions on the device behavior, which, for instance, may result in a reduced relative source resistance. The latter considers the entire device globally, including the combined effect from the access and trench regions, which may, in turn, hinder the local properties of the fins. For instance, a smaller $g_{\mathrm{m}}$ was observed in this paper because it was normalized by $w_{\text {footprint }}$. Normalizing device characteristics per footprint is more adapted when scaling up the devices and comparing the performance per cost of the technology.

The tri-gate does not always improve the linearity of $g_{\mathrm{m}}$ either, and such effect can depend on $w_{\text {fin }}$, as shown in Fig. 2(b). While devices with wider tri-gates did exhibit flatter $g_{\mathrm{m}}$ peaks than in planar devices, the full-width at half-maximum (FWHM) of the $g_{\mathrm{m}}$ was only about $2.9 \mathrm{~V}$ at $w_{\text {fin }}=123 \mathrm{~nm}$, which was much smaller than that of the planar device $(5.5 \mathrm{~V})$. A similar phenomenon has also been observed in [3], [4], and [14]. While the underlying mechanism for this observation is not yet fully clear, it is likely due to the significant increase in capacitance per fin area $\left(C_{\text {fin }}\right)$ with narrowing fins (which will be discussed later). An alternative approach to enhance the linearity is to integrate parallel fins with different $w_{\text {fin }}$ in a single tri-gate device [31]. Such multi$w_{\text {fin }}$ tri-gate transistors function as parallel transistors with different $V_{\mathrm{TH}}$, which can greatly enhance the linearity, thanks to the smooth dependence of $V_{\mathrm{TH}}$ on $w_{\text {fin }}$ in tri-gate $\mathrm{GaN}$ transistors [Fig. 2(c)]. In addition to such tri-gate technologies, planar nanostrip channels are also very promising to enhance the linearity of the device, as demonstrated in [34].

\section{IMPACT ON THRESHOLD VOLTAGE}

The dependence of $V_{\mathrm{TH}}$ on $w_{\text {fin }}$ [Fig. 2(c)] is one of the most important features of tri-gate $\mathrm{GaN}$ devices, which can be used to control the breakdown voltage, leakage current, as well as the linearity, and can be further adapted to form lateral $\mathrm{AlGaN} / \mathrm{GaN}$ field effect rectifiers [35] or current limiting diodes [36]. This dependence of $V_{\mathrm{TH}}$ on $w_{\text {fin }}$ is determined by several reasons, including the increased $C_{\text {fin }}$ and the reduced $N_{\text {s }}$ in narrower fins caused by strain relaxation and sidewall depletion, which can be described in more detail as follows.

1) Increased capacitance: a simplified model of a tri-gated fin includes three capacitors [inset of Fig. 2(c)]. The top capacitor $\left(C_{\text {top }}\right)$ is formed by the gate dielectric and the barrier layer, and the two sidewall capacitors $\left(2 \cdot C_{\text {side }}\right)$ are formed by the oxide and parts of the fin body. $C_{\text {side }}$ is a dynamic value that depends on both $w_{\text {fin }}$ and $V_{\mathrm{G}}$, and increases as $w_{\text {fin }}$ reduces, similar to double-gate junctionless transistors. It should be noted that the $C_{\text {top }}$ could also increase as $w_{\text {fin }}$ decreases due to the fringing electric field, especially when $w_{\text {fin }}$ approaches the thickness of the barrier and the dielectric layers.

2) Strain relaxation: the strained $\mathrm{AlGaN} / \mathrm{GaN}$ heterostructure starts to relax after the fin etching by elastic deformation [37]-[40], which is nonuniform [41] and depends on the dimension of the fins [42], partially relieving the strain and reducing the $N_{\mathrm{s}}$.

3) Sidewall depletion: carriers within the fins are depleted within a certain width $\left(w_{\text {dep }}\right)$ from the sidewalls toward
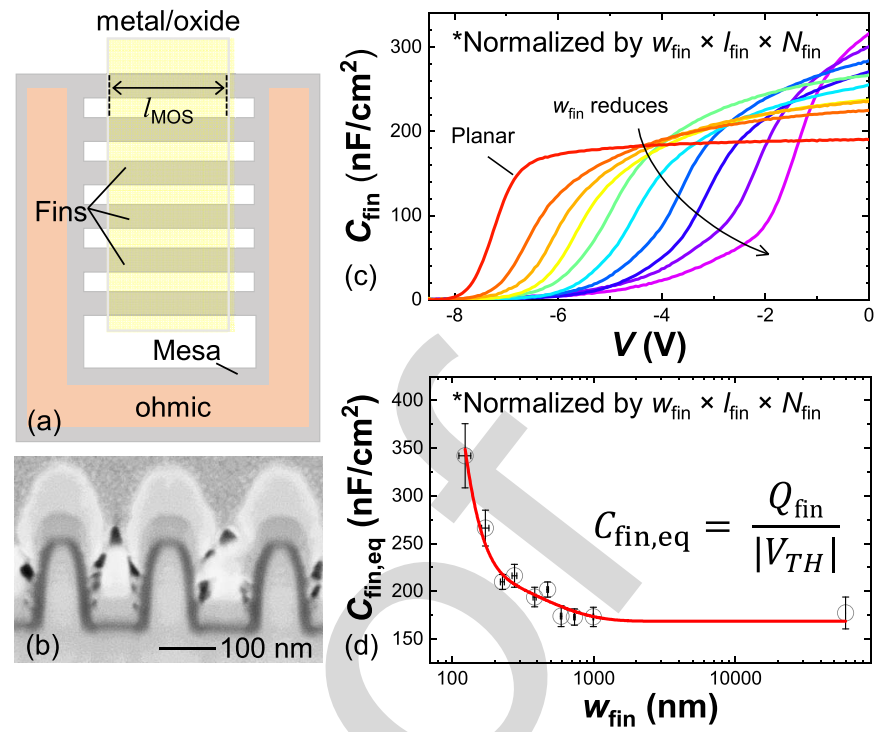

Fig. 3. (a) Schematic of the fabricated tri-gate MOS capacitor (b) Cross-sectional SEM image of a tri-gate MOS structure fabricated on a dummy sample, tilted by $52^{\circ}$. (c) Average capacitance per fin area $\left(C_{\text {fin }}\right)$ with different $w_{\text {fin }}$, measured at $1 \mathrm{MHz}$. (d) Calculated effective capacitance per fin area $\left(C_{\text {fin eq }}\right)$ versus $w_{\text {fin }}$. All capacitors had the same $w_{\text {footprint }}$ of $60 \mu \mathrm{m}$ and FF of 0.25 . The length of the anode (IMOS) metal was $3 \mu \mathrm{m}$ and the $I_{\text {fin }}$ was $4 \mu \mathrm{m}$. Capacitance values here were normalized by the top surface area of the fins $\left(w_{\text {fin }} \times I_{\text {MOS }} \times N_{\text {fin }}\right)$ to understand the control of the $2 D E G$ which was only in the fins.

the fin center [inset in Fig. 2(c)]. Thus $N_{\mathrm{s}}$, even at the center of the fin, can be affected by sidewall depletion, which is a crucial factor that allows achieving normallyOFF tri-gate GaN transistors. In addition, $w_{\text {dep }}$ is also important to determine the equivalent width of the 2DEG $\left(w_{2 \mathrm{DEG}}\right)$ within each fin and extract an equivalent $N_{\mathrm{S}}$.

We investigated these three factors to understand the $V_{\mathrm{TH}}-w_{\text {fin }}$ dependence in tri-gate $\mathrm{GaN}$ transistors. Tri-gate AlGaN/GaN MOS capacitors were fabricated [Fig. 3(a) and (b)], and their capacitance-voltage $(C-V)$ characteristics were measured [Fig. 3(c)], in which the $C_{\text {fin }}$ was normalized by the top surface area of the fins, namely $w_{\text {fin }} \times l_{\text {MOS }} \times N_{\text {fin }}$, in order to represent the control of the 2DEG, which is only present within the fins. The planar capacitor presented a nearly constant capacitance, which dropped sharply when $V$ reached $V_{\mathrm{TH}}$. In contrast, the depletion of tri-gate capacitors was progressive with applied $V . C_{\text {fin }}$ reduced gradually as $V$ approached $V_{\mathrm{TH}}$, with a steeper slope for smaller $w_{\text {fin }}$, since the sidewall gates progressively depleted the 2DEG from the sides to the center of the fin. Considering that $C_{\text {fin }}$ of the tri-gate varies with the voltage and is difficult to quantify, here we propose an effective $C_{\text {fin,eq }}=Q /\left|V_{\mathrm{TH}}\right|$, in which $Q$ was the charge determined from the integration of $C_{\text {fin }}$, to explore the dependence of tri-gate control on $w_{\text {fin }}$. As shown in Fig. 3(d), $C_{\text {fin,eq }}$ increased with narrowing fins, especially for $w_{\text {fin }}<200 \mathrm{~nm}$. When $w_{\text {fin }}=123 \mathrm{~nm}, C_{\text {fin,eq }}$ was as high as $342 \pm 34 \mathrm{nF} / \mathrm{cm}^{2}$, which is about twice the value of the planar capacitor $\left(177 \pm 17 \mathrm{nF} / \mathrm{cm}^{2}\right)$. This effect reflects the enhanced electrostatic control that contributed to the positive shift in $V_{\mathrm{TH}}$ with reduced $w_{\text {fin. }}$. Fig. $3(\mathrm{~d})$ offers general guidelines

"C 2019 IEEE. Personal use of this material is permitted. Permission from IEEE must be obtained for all other uses, in any current or future media, including reprinting/republishing this material for advertising or promotional purposes, creating new collective works, for resale or redistribution to servers or lists, or reuse of any copyrighted component of this work in other works. 

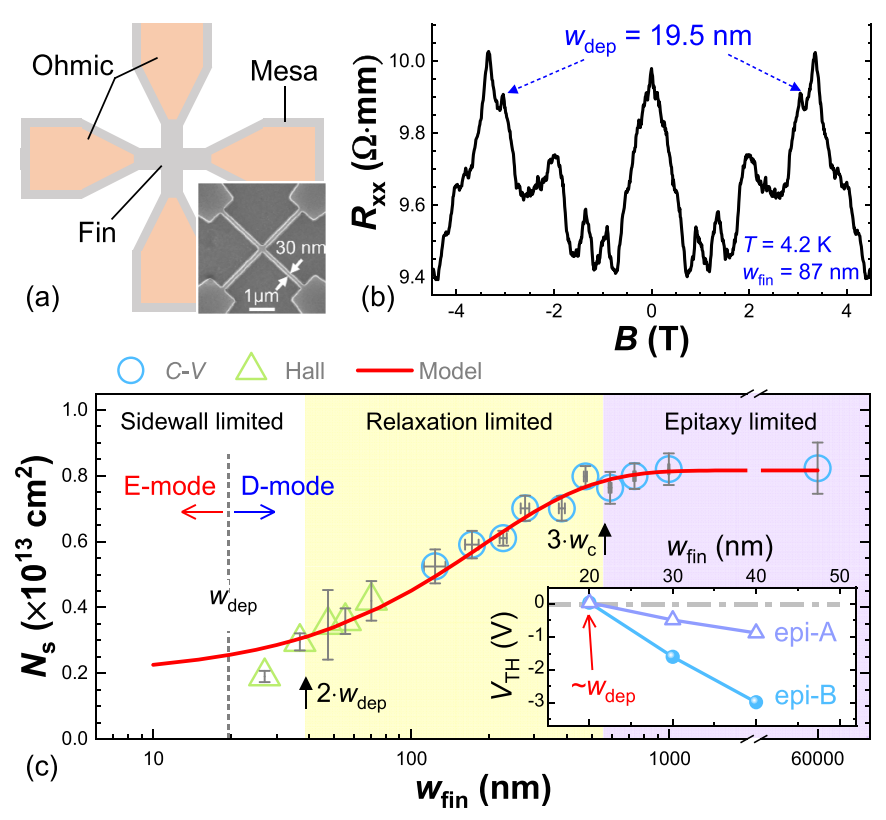

Fig. 4. (a) Schematic and SEM image of the fabricated hall cross. (b) $R_{\mathrm{xx}}$ versus magnetic field measured at $4.2 \mathrm{~K}$. (c) $N_{\mathrm{S}}$ versus $w_{\mathrm{fin}}$, extracted from both MOS capacitors and Hall crosses. The $N_{S}$ from $C-V$ measurements was normalized by $w_{2 D E G} \times I_{\text {MOS }} \times N_{\text {fin }}$, in which the $w_{2 D E G}$ was equal to $\left(w_{\text {fin }}-2 \cdot w_{\text {dep }}\right)$. The inset shows the $V_{T H}$ at $1 \mu \mathrm{A} / \mathrm{mm}$ in tri-gate GaN MOSHEMTs with $w_{\text {fin }}$ from 20 to $40 \mathrm{~nm}$ using different epi structures, in which epi-A and epi-B had $N_{\mathrm{S}}$ of $1 \times 10^{13} \mathrm{~cm}^{-2}$ and $1.5 \times 10^{13} \mathrm{~cm}^{-2}$, respectively.
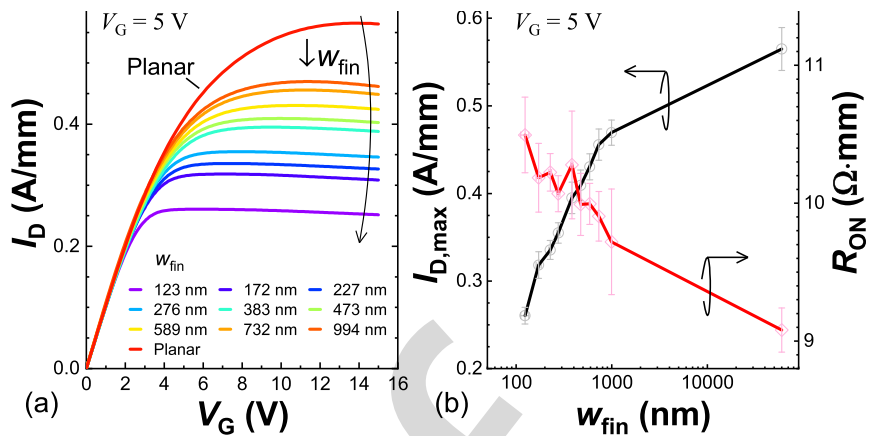

Fig. 5. (a) Average output characteristics of tri-gate and planar MOSHEMTs, measured at $V_{\mathrm{G}}=5 \mathrm{~V}$ and normalized by $w_{\text {footprint }}$. (b) Summarized dependences of $I_{D, \max }$ and $R_{\mathrm{ON}}$ on $w_{\mathrm{fin}}$.

without the oxide in a dummy sample, and the impact of the fixed charges for tri-gate capacitors with different $w_{\text {fin }}$ is not easy to be quantified.

The reduction in $N_{\mathrm{s}}$ from planar to narrow tri-gates can be analytically modeled as

$$
N_{\mathrm{s}, \text { fin }}=(1-\beta) \cdot N_{\mathrm{s}, \mathrm{p}}
$$

in which $N_{\mathrm{s} \text {, fin }}$ and $N_{\mathrm{s}, \mathrm{p}}$ are the carrier densities in $\mathrm{AlGaN} / \mathrm{GaN}$ fin and planar structures, respectively. $\beta\left(w_{\text {fin }}\right)$ describes the strain relaxation with reducing $w_{\text {fin }}$, and can be written as [37], [38], [42]

$$
\beta=\alpha \cdot e^{-w_{\text {fin }} / w_{\mathrm{c}}}
$$

which resulted in $\alpha=0.623$ and $w_{\mathrm{c}}=187 \mathrm{~nm}$ from fitting this model with the experimental data shown in Fig. 4(a). The values of $\alpha$ and $w_{\mathrm{c}}$ can be impacted by different fin heights, lengths, and epi structures. The quantity $w_{\mathrm{c}}$ represents a characteristic width for relaxation such that the $N_{\mathrm{s}}-w_{\text {fin }}$ dependence is relaxation-limited when $w_{\text {fin }}$ is below $3 \cdot w_{\mathrm{c}}$ [Fig. 4(c)]. When $w_{\text {fin }}$ is greater than $3 \cdot w_{\mathrm{c}}$, the effect of relaxation is minor, thus $N_{\mathrm{s}}$ is limited by the design and growth of the AlGaN/GaN heterostructure, varying little with $w_{\text {fin }}$. When $w_{\text {fin }}$ is smaller than $2 \cdot w_{\text {dep }}, N_{\mathrm{s}}$ is limited by the sidewall depletion, and reduces much faster than the model prediction, as the fins get narrow enough so that the sidewall depletion affects the $2 \mathrm{DEG}$ even at the center of the fin. This defines three main regions of the $N_{\mathrm{s}}-w_{\text {fin }}$ characteristics: sidewall-limited, relaxation-limited, and epitaxy-limited regions [Fig. 4(c)].

The sidewall depletion is a crucial factor to achieve normally-OFF operation, as it can even remove most of the 2DEG in the fins when $w_{\text {fin }}$ is sufficiently small ( $\leq w_{\text {dep }}$ ). As shown in the inset in Fig. 4(c), the normally-OFF operation could be achieved for $w_{\text {fin }}=20 \mathrm{~nm}\left(\approx w_{\text {dep }}\right)$ with $V_{\mathrm{TH}}$ of $0.04 \mathrm{~V}$ at $1 \mu \mathrm{A} / \mathrm{mm}$, regardless of different epi structures with different $N_{\mathrm{s}}$, indicating the effective depletion of the 2 DEG by the sidewalls. This value of $w_{\text {fin }}$ agrees well with previous reports showing that the effective nonconductive width in GaN fins was between 19 and $26 \mathrm{~nm}$ [44].

\section{IMPACT ON OUTPUT CHARACTERISTICS}

In addition to transfer characteristics, the ON-state performance of the device is also impacted by $w_{\text {fin }}$, as narrowing

"C 2019 IEEE. Personal use of this material is permitted. Permission from IEEE must be obtained for all other uses, in any current or future media, including reprinting/republishing this material for advertising or promotional purposes, creating new collective works, for resale or redistribution to servers or lists, or reuse of any copyrighted component of this work in other works. 
(a) Planar

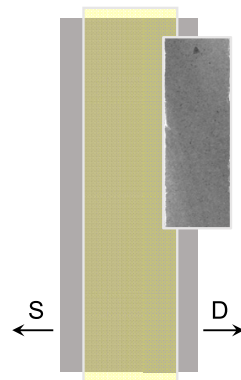

(b) Half-planar

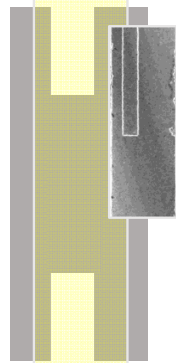

$w_{\text {channel }}=60 \mu \mathrm{m}$ $W_{\text {trench }}=0 \mu \mathrm{m}$

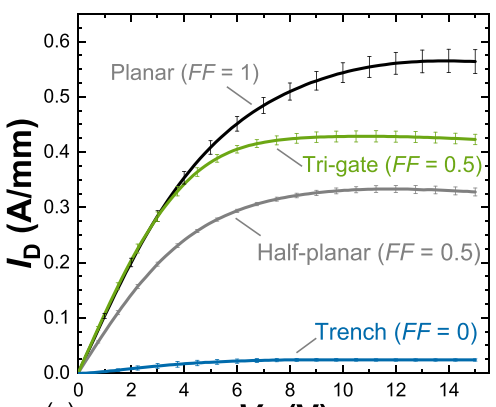

(e) (c) Tri-gate

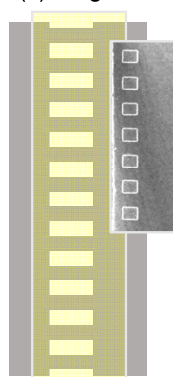

$w_{\text {channel }}=30 \mu \mathrm{m}$ $W_{\text {trench }}=30 \mu \mathrm{m}$ (d) Trench

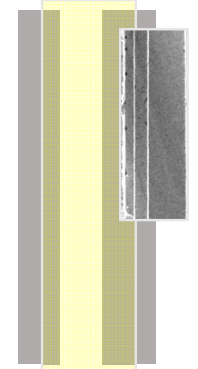

$W_{\text {channel }}=0 \mu \mathrm{m}$ $w_{\text {trench }}=60 \mu \mathrm{m}$

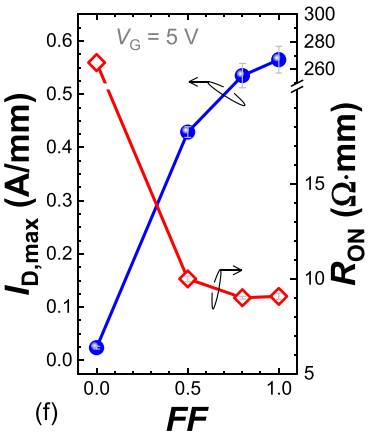

Fig. 6. (a)-(d) Top view schematic of the four types of transistors with similar dimensions but different gate regions. (e) Average output characteristics at $V_{G}=5 \mathrm{~V}$. (f) Dependences of $I_{D}$, max and $R_{\mathrm{ON}}$ on FF at $V_{\mathrm{G}}=5 \mathrm{~V}$.

the fins degrades the $I_{\mathrm{D}, \max }$ and $R_{\mathrm{ON}}$ [Fig. 5(a) and (b)]. $I_{\mathrm{D}, \max }$ reduced from $565 \pm 25 \mathrm{~mA} / \mathrm{mm}$ in the planar device to $261 \pm 10 \mathrm{~mA} / \mathrm{mm}$ in the tri-gate device with $w_{\text {fin }}$ of $123 \mathrm{~nm}$, corresponding to a significant reduction of $53.9 \%$. This is due to the narrower effective channel width $\left(w_{\text {channel }}=w_{\text {fin }} \times N_{\text {fin }}\right)$ in tri-gate devices and also the increased sidewall scattering in narrow fins. From planar to $w_{\text {fin }}=123 \mathrm{~nm}, R_{\mathrm{ON}}$ increased by $15.5 \%$, which is likely due to the degraded electron mobility and larger spreading resistance in narrower fins [45]. The degradation in $R_{\mathrm{ON}}$ was much less pronounced than in $I_{\mathrm{D}, \max }$, as the length of the etched region (the trenches) was small with respect to the length of the entire active region of the device, but $I_{\mathrm{D}}$ was greatly affected by the bottleneck on the current path from the narrow fins. The different $V_{\mathrm{TH}}$ in the devices was not considered here since the devices were all normally-ON and $V_{\mathrm{G}}=5 \mathrm{~V}$ was sufficiently large to nearly saturate their $R_{\mathrm{ON}}$.

When investigating the impact of $w_{\text {fin }}$ on $I_{\mathrm{D}, \max }$, and $R_{\mathrm{ON}}$, it is very important to isolate it from other factors, such as the effective source injection, the trench conduction, and the FF, since they all can influence the performance of the device. To illustrate the effect of effective source injection and trench conduction, we compared four types of transistors with similar dimensions but different gate regions.

1) The Planar [Fig. 6(a)] was a planar-gate MOSHEMT with $w_{\text {channel }}=w_{\text {footprint }}$ of $60 \mu \mathrm{m}$.

2) The Half-planar [Fig. 6(b)] had one 30- $\mu \mathrm{m}$ wide "fin" $\left(w_{\text {channel }}\right)$ and two $15-\mu \mathrm{m}$ wide trenches $\left(w_{\text {trench }}=\right.$ $30 \mu \mathrm{m}$ ) in its gate region, corresponding a FF of 0.5.
J) 1 IIC 111-gale [1'ly. U(C)] was a lli-gale IVIUNILivil will FF also of 0.5 , in which $600-n m$ wide fins were evenly distributed in the tri-gate region, resulting in $w_{\text {channel }}$ and $w_{\text {trench }}$ of both $30 \mu \mathrm{m}$, the same as the Half-planar.

4) The Trench [Fig. 6(d)] was similar to other devices except for its fully recessed gate region, resulting in $w_{\text {channel }}$ and $w_{\text {trench }}$ of 0 and $60 \mu \mathrm{m}$, respectively. The recess depth was the same as the fin height in the Tri-gate.

The output characteristics of these devices at $V_{\mathrm{G}}=5 \mathrm{~V}$ were measured and compared in Fig. 6(e), all normalized by $w_{\text {footprint }}$ of $60 \mu \mathrm{m}$. While the Half-planar had a $w_{\text {channel }}$ of $30 \mu \mathrm{m}$ (e.g., half of the channel width of the Planar), it exhibited $I_{\mathrm{D}, \max }$ of $333 \pm 6 \mathrm{~mA} / \mathrm{mm}$, which is larger than half of $I_{\mathrm{D}, \max }$ in Planar $(565 \pm 21 \mathrm{~mA} / \mathrm{mm})$. This is because the width of the effective source injection $\left(w_{\text {source }}\right)$ in the Half-planar was greater than its $w_{\text {channel }}$. Assuming a uniform injection from the source and ignoring the crowding effect, $w_{\text {source }}$ in Half-planar can be estimated using

$$
w_{\text {source }}=\frac{\left(I_{\mathrm{D}, \text { max }}^{\text {Half }- \text { planar }}-0.5 \times I_{\mathrm{D}, \max }^{\text {Planar }}\right) \times w_{\text {footprint }}}{I_{\mathrm{D}, \max }^{\text {Planar }}}
$$

which is $35.4 \mu \mathrm{m}$, indicating that the effective source extended $2.7 \mu \mathrm{m}$ from each side of the channel toward the mesa edge. This results in a larger $I_{\mathrm{D}, \max }$ ratio between the Half-planar and Planar $(58.9 \%)$ than the FF of 50\% of the Half-planar.

$I_{\mathrm{D}, \max }$ can be further enhanced by increasing $w_{\text {source }}$ for a fixed FF, by dividing the $30-\mu \mathrm{m}$ wide "fin" (Half-planar) into many narrower fins and distributing them evenly in the device (Tri-gate). As shown in Fig. 6(e), the Tri-gate presented much larger $I_{\mathrm{D}, \max }(429 \pm 10 \mathrm{~mA} / \mathrm{mm})$ than the Half-planar, although they had the same $w_{\text {channel }}$ and FF. This is due to the much larger $w_{\text {source }}$ in the Tri-gate, which can be comparable to that in the Planar as the spacing between the fins was $0.6 \mu \mathrm{m}$, which is much smaller than the extension length $(2.7 \mu \mathrm{m})$ of the effect source injection (here we assumed that $w_{\text {fin }}$ did not significantly change the extension length of the effective source).

The trenches also contributed to the ON-state conduction of the transistors, as they function as trench MOSFETs in parallel with the fin-MOSHEMTs, which can be seen from the $I_{\mathrm{D}}-V_{\mathrm{D}}$ characteristics of Trench shown in Fig. 6(e). The trench conduction is small in normally-ON transistors, due to the large difference in $V_{\mathrm{TH}}$ of these MOSFETs and MOSHEMTs, as well as the poor electron mobility of the trench MOSFETs, which is limited by the MOS interface and the damages caused by the trench etching [16]. However, it may not be negligible in normally-OFF tri-gate $\mathrm{GaN}$ transistors, depending on the design of the tri-gate region.

In addition to $w_{\text {source }}$ and trench conduction, FF is another important factor that should be fixed when comparing tri-gate transistors with different $w_{\text {fin }}$. As shown in Fig. 6(f), $I_{\mathrm{D} \text {,max }}$ in tri-gate devices was enhanced by increasing the FF, despite the same $w_{\text {fin }}$, thanks to the increased $w_{\text {channel }}$. Such increase was not linear, as $w_{\text {source }}$ was wider than $w_{\text {channel }}$. At an FF of 0.8 , the differences in $I_{\mathrm{D}, \max }$ and $R_{\mathrm{ON}}$ between the planar and tri-gate devices became very small, within the error bars,

"C 2019 IEEE. Personal use of this material is permitted. Permission from IEEE must be obtained for all other uses, in any current or future media, including reprinting/republishing this material for advertising or promotional purposes, creating new collective works, for resale or redistribution to servers or lists, or reuse of any copyrighted component of this work in other works. 


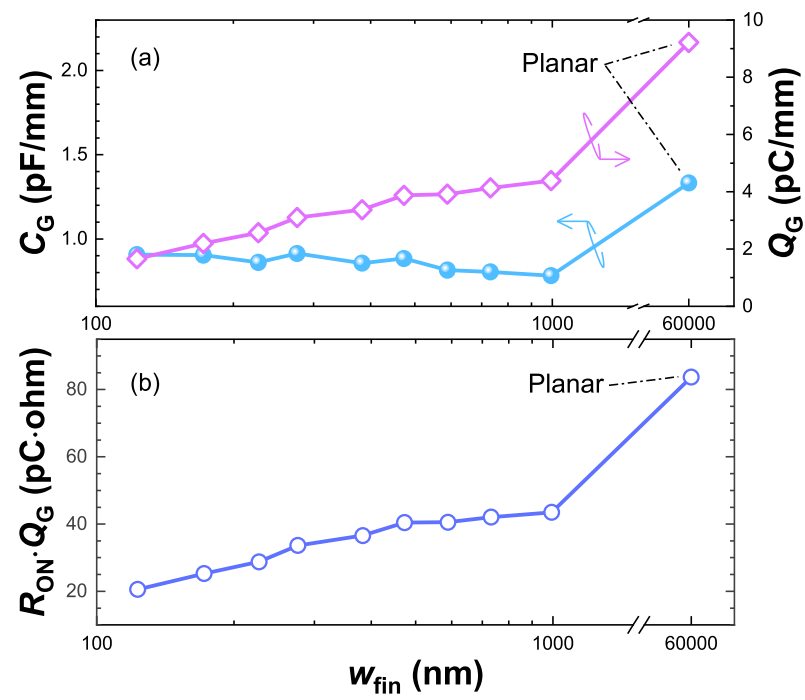

Fig. 7. (a) Dependence of $C_{\mathrm{G}}$ and $Q_{\mathrm{G}}$ on $w_{\text {fin }}$ normalized by $w_{\text {footprint }}$, extracted at $V_{G}=0 \mathrm{~V}$. (b) Dependence of $R_{\mathrm{ON}} \cdot Q_{\mathrm{G}}$ on $w_{\text {fin }}$. All results were extract at $V_{G}=0 \mathrm{~V}$ and $F F=0.5$.

revealing that the degradation caused by the etching of the 2DEG to form tri-gates was eliminated.

\section{IMPACT ON GATE CAPACITANCE AND CHARGE}

The tri-gate architecture can be an effective way to reduce $C_{\mathrm{G}}$ and $Q_{\mathrm{G}}$ in normally-ON GaN (MOS) HEMTs, depending on its design and $V_{\mathrm{G}}$. It is often considered that the tri-gate geometry increases the $C_{\mathrm{G}}$ due to its 3-D nature, as it is the case in FinFETs. This is true when $C_{\mathrm{G}}$ is evaluated for a single fin with small $w_{\text {fin }}$, since, in this case, the gate capacitance per area of 2DEG is indeed increased, as discussed in Fig. 3(d). However, it is usually more important to understand how the tri-gate impacts $C_{\mathrm{G}}$ and $Q_{\mathrm{G}}$ in the entire device footprint consisting of multiple parallel fins, especially for device designers on power and RF electronics. In this scenario, $C_{\mathrm{G}}$ and $Q_{\mathrm{G}}$ can be both greatly diminished in tri-gate compared to planar transistors, because a portion of the 2DEG carriers is removed during the fin etching, which is determined by FF, and only the carriers present at the 2-D channels in the fins contribute to the $C_{\mathrm{G}}$ and the $Q_{\mathrm{G}}$ (before the sidewall channels turn on). This is very different to FinFETs, in which the entire 3-D periphery of the fins contributes to the $C_{\mathrm{G}}$ and the $Q_{\mathrm{G}}$.

As shown in Fig. 7(a), the $C_{\mathrm{G}}$ of the tri-gate devices varied from 0.79 to $0.91 \mathrm{pF} / \mathrm{mm}$, depending on $w_{\text {fin }}$, which were between $58.6 \%$ and $68.4 \%$ of the planar device (at $\mathrm{FF}=0.5$ and $V_{\mathrm{G}}=0 \mathrm{~V}$ ). The $Q_{\mathrm{G}}$ was also reduced by over $50 \%$, resulting in a decreasing $R_{\mathrm{ON}} \cdot Q_{\mathrm{G}}$ product [Fig. 7(b)] and hence, a higher figure-of-merit for high-frequency switching.

These results indicate that, depending on its geometry and $V_{\mathrm{G}}$, the tri-gate can reduce $C_{\mathrm{G}}, Q_{\mathrm{G}}$, and $R_{\mathrm{ON}} \cdot Q_{\mathrm{G}}$ in normallyON GaN (MOS)HEMTs, which can be valuable for many applications.
$V_{\mathrm{TH}}, R_{\mathrm{ON}}, I_{\mathrm{D}, \max }, C_{\mathrm{G}}$, and $Q_{\mathrm{G}} . V_{\mathrm{TH}}$ was increased when $w_{\mathrm{fin}}$ was reduced, thanks to the larger $C_{\text {fin,eq }}$ and the smaller $N_{\mathrm{s}}$. A narrower fin degraded the $R_{\mathrm{ON}}$ and $I_{\mathrm{D}, \max }$, which, however, could be recovered by increasing the FF. Finally, the tri-gate reduced $C_{\mathrm{G}}$ and $Q_{\mathrm{G}}$ for normally-ON MOSHEMTs, which, however, depends on the $V_{\mathrm{G}}$ and the design of the tri-gate.

The dependence of these key characteristics upon $w_{\text {fin }}$ in tri-gated $\mathrm{GaN}$ heterostructures offers additional tools to tailor the performance of $\mathrm{GaN}$ electronic devices for various applications. In addition to achieving normally-OFF operation by decreasing $w_{\text {fin }}$, the dependence of $V_{\mathrm{TH}}$ on $w_{\text {fin }}$ makes the tri-gate technology very promising for new FPs for power GaN devices. While current FP technologies rely on vertical designs by tuning the thickness and the inclination of the dielectric to spread the electric field, tri-gates offer a lateral design approach for FPs that can be easily fabricated by merely tuning $w_{\text {fin }}$ lithographically [24]-[26]. In addition, the $V_{\mathrm{TH}}-w_{\text {fin }}$ dependence in tri-gate $\mathrm{GaN}$ devices is excellent to enhance the linearity in GaN RF HEMTs, by integrating several $w_{\text {fin }}$ in the tri-gate region, which can lead to a $20-\mathrm{dB}$ reduction in harmonics and a 6-dB enhancement in the output third-order intercept point, as demonstrated in [31].

The results revealed in this paper provide broader and further insights about tri-gate GaN devices, shedding light on several subtle points, and offering important understanding to design tri-gate GaN devices for different applications, which are fundamental for the further advance of tri-gate $\mathrm{GaN}$ technologies.

\section{ACKNOWLEDGMENT}

The authors would like to thank the staff in CMi and ICMP cleanrooms at EPFL for technical support.

\section{REFERENCES}

[1] J. Ma and E. Matioli, "High performance tri-gate GaN power MOSHEMTs on silicon substrate," IEEE Electron Device Lett., vol. 38, no. 3, pp. 367-370, Mar. 2017. doi: 10.1109/LED.2017.2661755.

[2] J. Ma and E. Matioli, "High-voltage and low-leakage AlGaN/GaN tri-anode Schottky diodes with integrated tri-gate transistors," IEEE Electron Device Lett., vol. 38, no. 1, pp. 83-86, Jan. 2017. doi: 10.1109/LED.2016.2632044.

[3] D. F. Brown, Y. Tang, D. Regan, J. Wong, and M. Micovic, "Selfaligned AlGaN/GaN FinFETs," IEEE Electron Device Lett., vol. 38, no. 10, pp. 1445-1448, Oct. 2017. doi: 10.1109/LED.2017.2747843.

[4] S. Liu et al., "Enhancement-mode operation of nanochannel array (NCA) AlGaN/GaN HEMTs," IEEE Electron Device Lett., vol. 33, no. 3, pp. 354-356, Mar. 2012, doi: 10.1109/LED.2011.2179003.

[5] M. Azize et al., "High-electron-mobility transistors based on InAlN/GaN nanoribbons," IEEE Electron Device Lett., vol. 32, no. 12, pp. 1680-1682, Dec. 2011. doi: 10.1109/LED.2011.2170149.

[6] K. Im et al., "Normally off single-nanoribbon $\mathrm{Al}_{2} \mathrm{O}_{3} / \mathrm{GaN}$ MISFET," IEEE Electron Device Lett., vol. 34, no. 1, pp. 27-29, Jan. 2013. doi: 10.1109/LED.2012.2222861

[7] K. Ohi, J. T. Asubar, K. Nishiguchi, and T. Hashizume, "Current stability in multi-mesa-channel AlGaN/GaN HEMTs," IEEE Trans. Electron Devices, vol. 60, no. 10, pp. 2997-3004, Oct. 2013. doi: 10.1109/TED.2013.2266663.

[8] J. Ma, M. Zhu, and E. Matioli, "900 V reverse-blocking GaNon-Si MOSHEMTs with a hybrid tri-anode Schottky drain," IEEE Electron Device Lett., vol. 38, no. 12, pp. 1704-1707, Dec. 2017. doi: 10.1109/LED.2017.2761911.

[9] T. Wang, J. Ma, and E. Matioli, "1100 V AlGaN/GaN MOSHEMTs with integrated tri-anode freewheeling diodes," IEEE Electron Device Lett., vol. 39, no. 7, pp. 1038-1041, Jul. 2018. doi: 10.1109/LED.2018.2842031.

"C 2019 IEEE. Personal use of this material is permitted. Permission from IEEE must be obtained for all other uses, in any current or future media, including reprinting/republishing this material for advertising or promotional purposes, creating new collective works, for resale or redistribution to servers or lists, or reuse of any copyrighted component of this work in other works. 
[10] S. Takashima, Z. Li, and T. P. Chow, "Sidewall dominated characteristics on fin-gate AlGaN/GaN MOS-channel-HEMTs," IEEE Trans. Electron Devices, vol. 60, no. 10, pp. 3025-3031, Oct. 2013. doi: 10.1109/TED.2013.2278185.

[11] M. Alsharef, R. Granzner, and F. Schwierz, "Theoretical investigation of trigate AlGaN/GaN HEMTs," IEEE Trans. Electron Devices, vol. 60 , no. 10, pp. 3335-3341, Oct. 2013. doi: 10.1109/TED.2013.2279264.

[12] C. Yadav, P. Kushwaha, S. Khandelwal, J. P. Duarte, Y. S. Chauhan, and $\mathrm{C}$. $\mathrm{Hu}$, "Modeling of GaN-based normally-off FinFET," IEEE Electron Device Lett., vol. 35, no. 6, pp. 612-614, Jun. 2014. doi: 10.1109/LED.2014.2314700.

[13] S. Arulkumaran et al., " $\mathrm{In}_{0.17} \mathrm{Al}_{0.83} \mathrm{~N} / \mathrm{AlN} / \mathrm{GaN}$ triple T-shape finHEMTs with $g_{\mathrm{m}}=646 \mathrm{mS} / \mathrm{mm}, I_{\mathrm{ON}}=1.03 \mathrm{~A} / \mathrm{mm}, I_{\mathrm{OFF}}=1.13 \mu \mathrm{A} / \mathrm{mm}$, $\mathrm{SS}=82 \mathrm{mV} / \mathrm{dec}$ and $\mathrm{DIBL}=28 \mathrm{mV} / \mathrm{V}$ at $V_{D}=0.5 \mathrm{~V}$," in IEDM Tech. Dig., San Francisco, CA, USA, Dec. 2014, pp. 25.6.1-25.6.4. doi: 10.1109/IEDM.2014.7047109.

[14] M. Alsharef et al., "RF performance of trigate GaN HEMTs," IEEE Trans. Electron Devices, vol. 63, no. 11, pp. 4255-4261, Nov. 2016. doi: 10.1109/TED.2016.2606701.

[15] S. Vodapally et al., "1/f-noise in AlGaN/GaN nanowire omegaFinFETs," IEEE Electron Device Lett., vol. 38, no. 2, pp. 252-254, Feb. 2017. doi: 10.1109/LED.2016.2645211.

[16] J. Ma, G. Santoruvo, P. Tandon, and E. Matioli, "Enhanced electrical performance and heat dissipation in AlGaN/GaN Schottky barrier diodes using hybrid tri-anode structure," IEEE Trans. Electron Devices, vol. 63, no. 9, pp. 3614-3619, Jul. 2016. doi: 10.1109/TED.2016.2587801.

[17] E. Matioli, B. Lu, and T. Palacios, "Ultralow leakage current AlGaN/GaN Schottky diodes with 3-D anode structure," IEEE Trans. Electron Devices, vol. 60, no. 10, pp. 3365-3370, Oct. 2013. doi: 10.1109/TED.2013.2279120.

[18] K. Ren, Y. C. Liang, and C.-F. Huang, "Compact physical models for AlGaN/GaN MIS-FinFET on threshold voltage and saturation current," IEEE Trans. Electron Devices, vol. 65, no. 4, pp. 1348-1354, Apr. 2018 doi: 10.1109/TED.2018.2809517.

[19] Y. Xu, S. Cristoloveanu, M. Bawedin, K.-S. Im, and J.-H. Lee, "Performance improvement and sub- $60 \mathrm{mV} /$ decade swing in $\mathrm{AlGaN} / \mathrm{GaN}$ FinFETs by simultaneous activation of 2DEG and sidewall MOS channels," IEEE Trans. Electron Devices, vol. 65, no. 3, pp. 915-920, Mar. 2018. doi: 10.1109/TED.2017.2788920.

[20] M. Zhang et al., "Influence of fin configuration on the characteristics of AlGaN/GaN fin-HEMTs," IEEE Trans. Electron Devices, vol. 65, no. 5, pp. 1745-1752, May 2018. doi: 10.1109/TED.2018.2819178.

[21] J. Ma, C. Erine, P. Xiang, K. Cheng, and E. Matioli, "Multi-channel tri-gate normally-on/off AlGaN/GaN MOSHEMTs on Si substrate with high breakdown voltage and low ON-resistance," Appl. Phys. Lett., vol. 113, Dec. 2018, Art. no. 242102. doi: 10.1063/1.5064407.

[22] B. Lu, E. Matioli, and T. Palacios, "Tri-gate normally-off GaN power MISFET," IEEE Electron Device Lett., vol. 33, no. 3, pp. 360-362, Mar. 2012. doi: 10.1109/LED.2011.2179971.

[23] H. Zhou, X. Lou, S. B. Kim, K. D. Chabak, R. G. Gordon, and D. Y. Peide, "Enhancement-mode AlGaN/GaN fin-MOSHEMTs on $\mathrm{Si}$ substrate with atomic layer epitaxy $\mathrm{MgCaO}$," IEEE Electron Device Lett., vol. 38, no. 9, pp. 1294-1297, Sep. 2017. doi: 10.1109/LED.2017.2731993.

[24] J. Ma and E. Matioli, "Slanted tri-gates for high-voltage GaN power devices," IEEE Electron Device Lett., vol. 38, no. 9, pp. 1305-1308, Sep. 2017. doi: 10.1109/LED.2017.2731799.

[25] J. Ma and E. Matioli, "2 kV slanted tri-gate GaN-on-Si Schottky barrier diodes with ultra-low leakage current," Appl. Phys. Lett., vol. 112, pp. 052101-1-052101-4, Jan. 2018. doi: 10.1063/1. 5012866.

[26] J. Ma, D. C. Zanuz, and E. Matioli, "Field plate design for low leakage current in lateral GaN power Schottky diodes: Role of the pinch-off voltage," IEEE Electron Device Lett., vol. 38, no. 9, pp. 1298-1301, Sep. 2017. doi: 10.1109/LED.2017.2734644.
[27] J. Ma, G. Kampitsis, P. Xiang, K. Cheng, and E. Matioli, "Multichannel Tri-gate GaN power Schottky diodes with low ON-resistance," IEEE Electron Device Lett., vol. 40, no. 2, pp. 275-278, Feb. 2019 doi: 10.1109/LED.2018.2887199.

[28] Y.-W. Jo et al., "AlGaN/GaN FinFET with extremely broad transconductance by side-wall wet etch," IEEE Electron Device Lett., vol. 36, no. 10, pp. 1008-1010, Oct. 2015. doi: 10.1109/LED.2015.2466096.

[29] K. Zhang et al., "High-linearity AlGaN/GaN FinFETs for microwave power applications," IEEE Electron Device Lett., vol. 38, no. 5, pp. 615-618, May 2017. doi: 10.1109/LED.2017.2687440.

[30] D. S. Lee et al., "Nanowire channel InAlN/GaN HEMTs with high linearity of $g_{\mathrm{m}}$ and $f_{\mathrm{T}}$," IEEE Electron Device Lett., vol. 34, no. 8, pp. 969-971, Jun. 2013. doi: 10.1109/LED.2013.2261913.

[31] S. Joglekar, U. Radhakrishna, D. Piedra, D. Antoniadis, and T. Palacios, "Large signal linearity enhancement of AlGaN/GaN high electron mobility transistors by device-level $\mathrm{Vt}$ engineering for transconductance compensation," in IEDM Tech. Dig., San Francisco, CA, USA, Dec. 2017 pp. 25.3.1-25.3.4. doi: 10.1109/IEDM.2017.8268457.

[32] Y.-W. Jo et al., "Control of transconductance in high performance AlGaN/GaN FinFETs," in Proc. IEEE 11th Int. Conf. Power Electron. Drive Syst., Sydney, NSW, Australia, Jun. 2015, pp. 684-686. doi: 10.1109/PEDS.2015.7203514.

[33] C.-Y. Chen and Y.-R. Wu, "Studying the short channel effect in the scaling of the AlGaN/GaN nanowire transistors," J. Appl. Phys., vol. 113 , Jun. 2013, Art. no. 214501. doi: 10.1063/1.4808241

[34] W. Xing, Z. Liu, H. Qiu, G. I. Ng, and T. Palacios, "Planar-nanostripchannel InAlN/GaN HEMTs on Si With Improved $g_{\mathrm{m}}$ and $f_{\mathrm{T}}$ linearity," IEEE Electron Device Lett., vol. 38, no. 5, pp. 619-622, May 2017. doi: 10.1109/LED.2017.2689810

[35] C. Zhou, W. Chen, E. L. Piner, and K. J. Chen, "AlGaN/GaN dualchannel lateral field-effect rectifier with punchthrough breakdown immunity and low on-resistance," IEEE Electron Device Lett., vol. 31, no. 1, pp. 5-7, Jan. 2010. doi: 10.1109/LED.2009.2034761.

[36] A. Zhang et al., "AlGaN/GaN lateral CRDs with hybrid trench cathodes," IEEE Trans. Electron Devices, vol. 65, no. 6, pp. 2660-2665, Jun. 2018 doi: 10.1109/TED.2018.2822834.

[37] M. Azize and T. Palacios, "Top-down fabrication of AlGaN/GaN nanoribbons," Appl. Phys. Lett., vol. 98, no. 4, pp. 042103-1-042103-3, Jan. 2011. doi: 10.1063/1.3544048

[38] W. J. Tseng et al., "Strain relaxation in GaN nanopillars," Appl. Phys. Lett., vol. 101, Dec. 2012, Art. no. 253102. doi: 10.1063/1.4772481.

[39] S. Luryi and E. Suhir, "New approach to the high quality epitaxial growth of lattice-mismatched materials," Appl. Phys. Lett., vol. 49, p. 140, May 1986. doi: 10.1063/1.97204

[40] M. Hugues et al., "Strain evolution in GaN nanowires: From free-surface objects to coalesced templates," J. Appl. Phys., vol. 114, Aug. 2013 Art. no. 084307. doi: 10.1063/1.4818962.

[41] S. Arulkumaran et al., "Electron velocity of $6 \times 10^{7} \mathrm{~cm} / \mathrm{s}$ at $300 \mathrm{~K}$ in stress engineered InAlN/GaN nano-channel high-electron-mobility transistors," Appl. Phys. Lett., vol. 106, Jan. 2015, Art. no. 053502. doi: $10.1063 / 1.4906970$

[42] E. Ertekin, P. A. Greaney, and D. C. Chrzan, "Equilibrium limits of coherency in strained nanowire heterostructures," J. Appl. Phys., vol. 97, Jun. 2005, Art. no. 114325. doi: 10.1063/1.1903106.

[43] G. Santoruvo, A. Allain, D. Ovchinnikov, and E. Matioli, "Magnetoballistic transport in GaN nanowires," Appl. Phys. Lett., vol. 109, no. 10 , Sep. 2016, Art. no. 103102. doi: 10.1063/1.4962332.

[44] E. Matioli and T. Palacios, "Room-temperature ballistic transport in III-nitride heterostructures," Nano Lett., vol. 15, no. 2, pp. 1070-1075, Feb. 2015. doi: 10.1021/n1504029r.

[45] A. Dixit, A. Kottantharayil, N. Collaert, M. Goodwin, M. Jurczak, and $\mathrm{K}$. De Meyer, "Analysis of the parasitic S/D resistance in multiple-gate FETs," IEEE Trans. Electron Devices, vol. 52, no. 6, pp. 1132-1140, Jun. 2005. doi: 10.1109/TED.2005.848098. 